\title{
Nanoparticle Chemical Characterization
}

National Cancer Institute

\section{Source}

National Cancer Institute. Nanoparticle Chemical Characterization. NCI Thesaurus. Code C62334.

Analyses to determine the chemical properties of a nanoparticle. These chemical elements are requisites for molecular structure, and they determine both the reactivity and selectivity of nanoparticles. 